\title{
Cryogenic Operation and Testing of the Extended LHC Prototype Magnet String
}

A. Bézaguet, J. Casas-Cubillos, H. Guinaudeau, B. Hilbert, Ph. Lebrun, L. Serio, A. Suraci and R. van Weelderen

\begin{abstract}
After the assembly, commissioning and successful first operation of a full-scale superconducting magnet string, and as a new prototype dipole magnet was added to approach final configuration, the cryogenic system has been slightly modified to allow the verification of the performance of the superfluid helium cooling loop in counter-current two-phase flow. At the same time the control system strategies have been updated and only two quench relief valves have been installed, one at each end of the string. We report on the cryogenic operation of the extended version of the string and the response of the system to transients.
\end{abstract}

LHC Division, CERN, CH-1211 Geneva 23, Switzerland

ICEC 16/ICMC, Kitakyushu, Japan, 20-24 May 1996

Administrative Secretariat

LHC Division

CERN

$\mathrm{CH}$ - 1211 Geneva 23

Switzerland

Geneva, 16 July, 1996 
Cryogenic Operation and Testing of the Extended LHC Prototype Magnet String

A. Bézaguet, J. Casas-Cubillos, H. Guinaudeau, B. Hilbert, Ph. Lebrun,

L. Serio, A. Suraci and R. van Weelderen

LHC Division, CERN, CH-1211 Geneva 23, Switzerland

\begin{abstract}
After the assembly, commissioning and successful first operation of a full-scale superconducting magnet string, and as a new prototype dipole magnet was added to approach final configuration, the cryogenic system has been slightly modified to allow the verification of the performance of the superfluid helium cooling loop in counter-current two-phase flow. At the same time the control system strategies have been updated and only two quench relief valves have been installed, one at each end of the string. We report on the cryogenic operation of the extended version of the string and the response of the system to transients.
\end{abstract}

\title{
INTRODUCTION
}

A major milestone in the validation of the basic technical choices for the Large Hadron Collider (LHC) project [1], as far as main accelerator systems - magnets, cryogenics and vacuum - are concerned, is the testing and operation of a full-scale superconducting magnet string, representing a half-cell of the machine lattice.

The first version of the LHC test string was assembled, commissioned and successfully operated for the first time at the end of 1994 [2], confirming the basic design choices of the LHC cryogenic system [3].

We report on the cryogenic operation of an extended and modified version of the string and in particular the counter-current flow cooling, the response to transients such as current ramp and discharge, as well as magnet resistive transitions.

\section{EXTENSION AND MODIFICATION OF THE STRING}

As a new prototype dipole magnet was added to lengthen the string towards its final configuration, the cryogenic system has been slightly modified to allow the verification of the performance of the superfluid helium cooling loop in counter-current two-phase flow, an option which would greatly simplify the cryogenic layout of the LHC machine.

The main modification was the introduction of a Joule-Thomson valve, a phase separator from which liquid overflows down the $1.4 \%$ slope, and a connection to the pumping line at the higher end of the string as shown in figure 1. In this configuration, pumping is shared between both ends of the helium II heat exchanger tube. Pumping from the higher end dominates because of pressure drop in the heat exchanger tube. The liquid and gas velocities are opposite one another giving rise to flow instabilities above certain flow rates.

At the same time the control system strategies have been updated profiting from the operational experience gained during the first run, in order to prepare the cryogenic system for the forthcoming experiment, that will validate the long term performance of the system during uninterrupted current cycling at nominal LHC rates. 
In line with previous results, only two quench relief valves have been installed, one at each end of the string. In this fashion, the quench relief valve scheme proposed for the LHC can be better approximated, and the requirements for the relief valves validated.

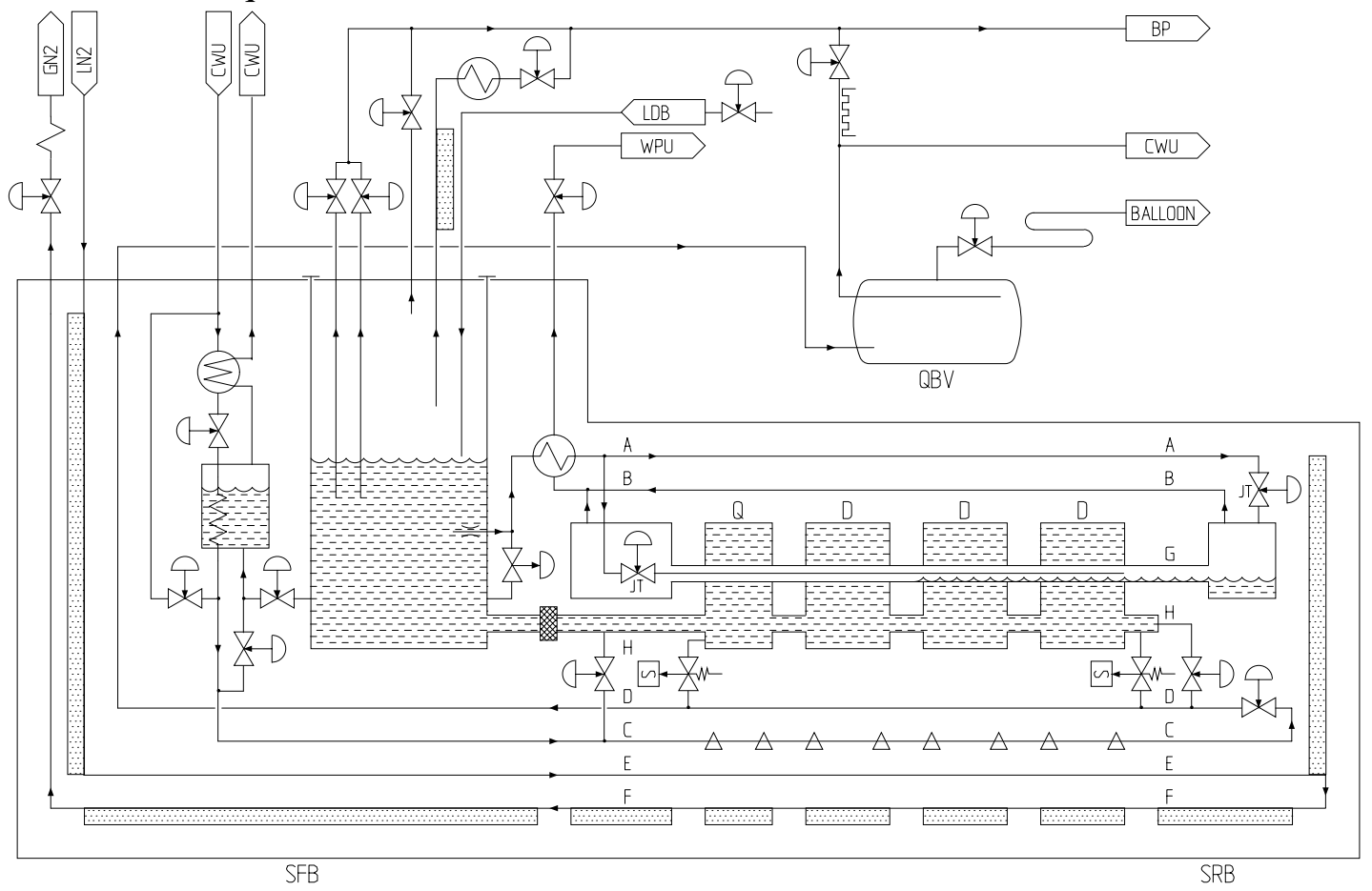

Fig. 1 Cryogenic flow-scheme of extended and modified string

\section{OPERATIONAL PERFORMANCE}

During 1995 and the beginning of 1996 the cryogenic system has reliably provided the nominal cryogenic conditions necessary to perform experiments concerning the main systems of the LHC, operating for more than 1500 hours at $1.9 \mathrm{~K}$ and recovering from more than 50 resistive transitions.

The temperature increase in the pressurised helium II bath was limited to $6 \mathrm{mK}$ during nominal current ramping $(10 \mathrm{~A} / \mathrm{s})$ and $50 \mathrm{mK}$ during nominal discharge $(-130 \mathrm{~A} / \mathrm{s})$, with steady-state temperature stability at $1.9 \mathrm{~K}$ better than a few $\mathrm{mK}$, both in co-current and counter-current operation of the saturated helium II loop.

In spite of the limitations reached in counter-current mode, the capability of cooling down the string from ambient temperature to $1.9 \mathrm{~K}$ in just more than a week has been maintained.

The operational experience gained during the first run has allowed us to define control strategies such that all stand-by and transient operations can be performed unattended in fully automatic mode. In the same way the magnet powering does not require presence of skilled cryogenic operator.

Opportunity was taken to perform other cryogenic test, such as the validation of basic instrumentation for LHC and the assessment of heat loads at different temperature levels.

\section{COUNTER-CURRENT FLOW OF TWO-PHASE SUPERFLUID HELIUM}

Since the superfluid helium cooling loop is now fed by overflow from the phase separator (figure 2), acting as a weir at the upper end, the neighboring section of the corrugated heat exchanger tube sees counter-current flow of liquid and vapor phases at saturation. As the heat load, and thus the mass flowrates are gradually increased, the velocity of the vapor flow rises up to the point where it transfers sufficient momentum to block the gravity-driven, counter-current liquid flow, and thus prevents wetting of the heat exchanger tube. As the conditions of flow blocking are approached, the liquid level in the phase separator must sharply rise to compensate for the decrease in liquid velocity, and an instability 
develops. This phenomenon has been extensively studied in vertical or quasi-vertical flows of conventional boiling fluids, and is known as "flooding" in boiler tubes [4], but no experimental work had been done on superfluid helium in quasi-horizontal geometries.

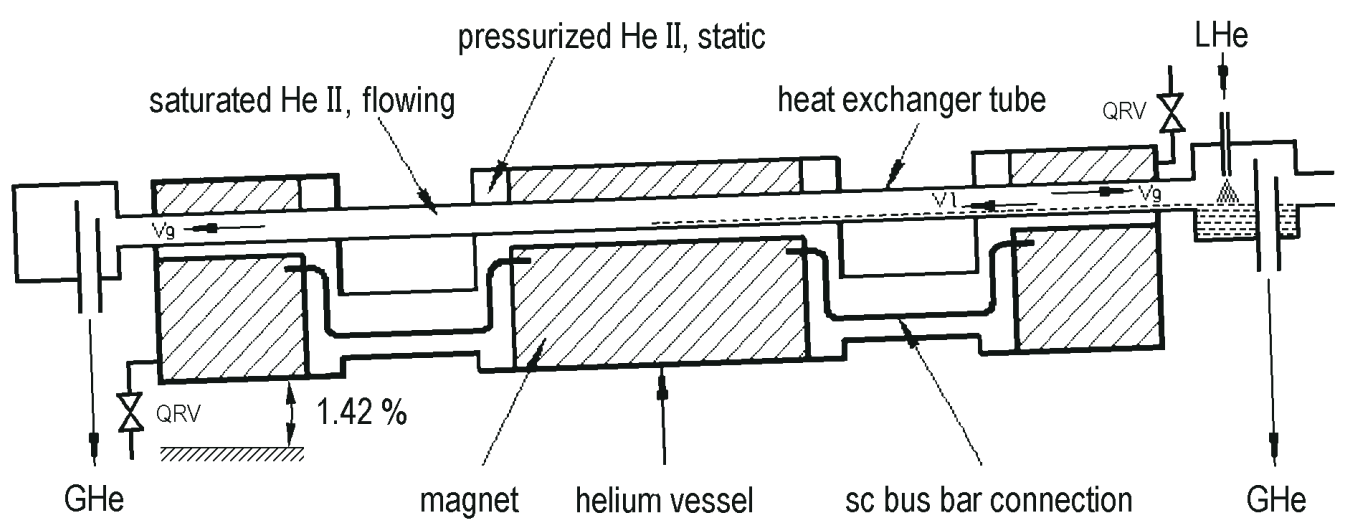

Fig.2 Superfluid helium cooling loop

In order to assess limitations to the superfluid helium cooling loop brought by this effect, experiments were performed by increasing heat loads, while maintaining magnet temperatures stable, at different values of saturation temperature (i.e. vapor density) between 4.5 and $1.8 \mathrm{~K}$. The onset of flow blockage can be clearly diagnosed from the sharp rise in the phase separator level, and the subsequent drift in the magnet temperatures (figure 3). The most critical conditions occur at low temperature, i.e. when the vapor density is lowest. On the $1.4 \%$ slope, the $1.8 \mathrm{~K}$ liquid flow in the 4.15 -cm inner-diameter corrugated tube is blocked at a vapor velocity of $6 \mathrm{~m} / \mathrm{s}$, corresponding to a heat load of $46 \mathrm{~W}$, i.e. above the nominal demand of the LHC half-cells [3]. Although occurring at higher mass flow-rate between 4.5 and $2.2 \mathrm{~K}$, blocking of the liquid flow in the heat exchanger tube may increase time for cool-down of the string from $4.5 \mathrm{~K}$. Strategy for fast cool-down is to optimize the saturation conditions as a function of magnet temperature, in order to work with high-density, low velocity vapor, while preserving sufficient driving temperature difference. In this fashion, cool-down from 4.5 to $1.8 \mathrm{~K}$ could be achieved in 6 hours, only slightly longer than Joule-Thomson valve sizing could permit.

\section{MAGNET RESISTIVE TRANSITIONS}

Coping with resistive transitions in superconducting magnets is a concern of main importance for the safe operation of the LHC. When occurring, it provokes the discharge of the magnetic energy stored in the coils (several MJ) into the static liquid helium bath, provoking rapid thermohydraulic transients

At a current of $13.1 \mathrm{kA}$, corresponding to the LHC ultimate magnetic field of $9 \mathrm{~T}$, rates of pressure increase up to $140 \mathrm{bar} / \mathrm{s}$ and pressure peaks reaching $12 \mathrm{bar}$ are commonly observed.

A fast responding protection system was designed at CERN to trigger electrically the opening of two quench relief valves after $120 \mathrm{~ms}$, with full opening less than $170 \mathrm{~ms}$ after quench detection. During the latest experimental run, it was shown that one quench relief valve, located either on the lower part and low end of the cold mass (discharging liquid helium) or on its upper part and high end (discharging mainly in gaseous or supercritical phase) is sufficient to prevent the 1000 litres of the magnet helium inventory from reaching the design pressure of 20 bar.

As shown in figure 4 the opening of the quench relief valve located on the upper part of the cold mass (the other being mechanically blocked) was delayed, in step of $10 \mathrm{~ms}$, up to a total delay of $200 \mathrm{~ms}$. In the dipoles (where the rate of pressure increase are the highest), the pressure peak increased by 2 bar with respect to no delay, still well below the 20 bar design pressure of the cold mass vessel. While keeping the maximum delay time for the electrical trigger, we lowered the pressure force on the valve seat down to 10 bar in order to force the valve to open on pressure. The pressure actuated opening gave a total delay time of $165 \mathrm{~ms}$ and a cold mass peak pressure of 13.5 bar.

These tests were aimed at investigating the use of modified commercial valves in order to avoid the use of specially designed, fast-opening prototype valves as the ones in use [5]. 
The final tests were done using a standard and commercially available industrial valve, modified to fully open in less than $300 \mathrm{~ms}$, mounted on the upper part and high end of the cold mass as one of the two fast opening valves. The test of this valve showed comparable discharge rates and pressure peaks in the cold mass.

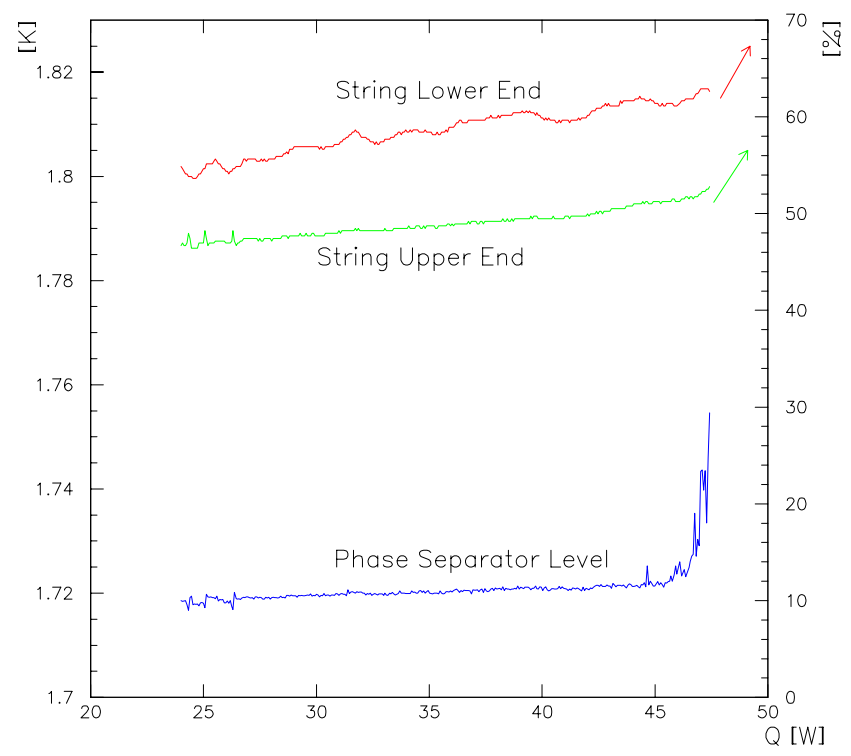

Fig.3 Flow instability test performed at $1.8 \mathrm{~K}$

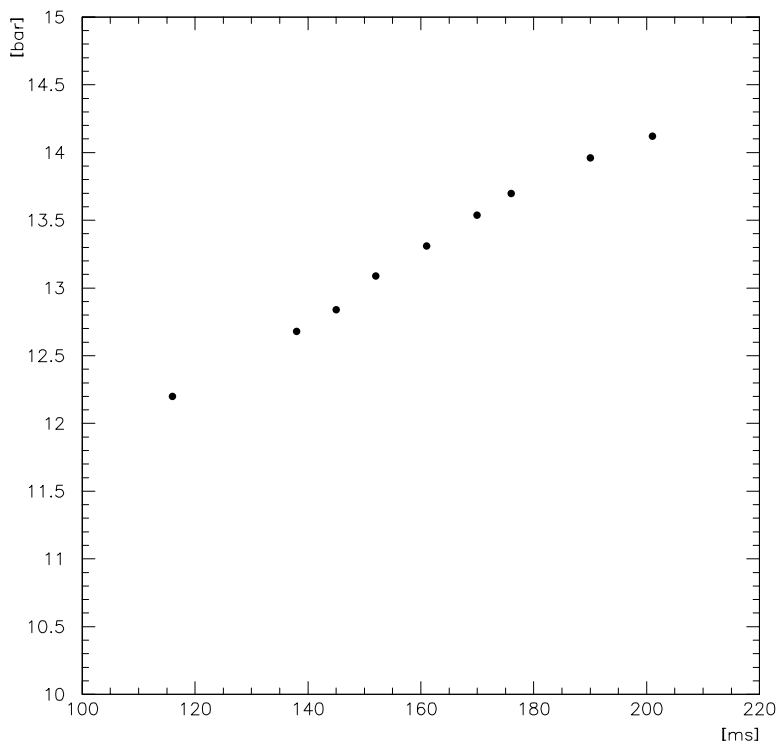

Fig.4 Cold mass peak pressure versus opening time

\section{CONCLUSION}

The basic design technical choices for LHC and the modifications introduced after the first experimental tests have been validated, giving us confidence on the cryogenic system that is going to be built for LHC.

The practical limitations of the counterflow system have been investigated and will give useful information for the definition of the final design which respect operational constraints.

The tests performed on the prototype quench relief valves have given decisive information for the specification of the quench relief valves to be used in LHC. Analysis and simulation work is under way to understand the physics behind the process of energy transfer to the helium bath after a quench and its subsequent discharge and recovery.

The string is now being prepared to assure 3000 hours of uninterrupted and fully automated operation at $1.9 \mathrm{~K}$ with continuous ramping and de-ramping to and from nominal current, to gain information on long-term behaviour of key components over the complete lifetime of the future machine.

\section{ACKNOWLEDGEMENTS}

The authors wish to thank the LHC String Team, B. Gaillard-Grenadier and Th. Goiffon for the valuable work performed during the operation and maintenance of the LHC test string.

\section{REFERENCES}

1. Evans, L.R., The Large Hadron Collider Project, paper presented at this conference

2 BÈzaguet, A., Casas-Cubillos, J., Flemsaeter, B., Gaillard-Grenadier, B., Goiffon, Th., Guinaudeau, H., Lebrun, Ph., Marquet, M., Serio, L., Suraci, A., Tavian, L. and van Weelderen, R., The superfluid helium cryogenic system for the LHC test string: design, construction and first operation, paper presented at CEC 1995, Columbus (1995) 
3. Lebrun, Ph., Superfluid helium cryogenics for the Large Hadron Collider Project, Cryogenics ICEC Supplement (1994) 34 1-8

4. Wallis, G.B., One-dimensional two-phase flow, McGraw-Hill (1969)

5. Danielsson, H., Ferlin, G., Jenninger, B, Luguet, C., Milner, S.E. and Rieubland, J.M., Cryogenic performance of a superfluid helium relief valve for the LHC superconducting magnets, paper presented at CEC 1995, Columbus (1995) 